\title{
Environment Education-An Example of a Comprehensive Experiment of Preparing Drilling Fluid Using Guar Gum Fracturing Backflow Fluid
} Gang $\mathrm{CHEN}^{1,{ }^{*}}$, Fan $\mathrm{ZHANG}^{1}$, Chao $\mathrm{CHEN}^{1}$, Jie $\mathrm{ZHANG}^{1}$, Dan XUE
Shi-jun $\mathrm{CHEN}^{1,3}$

${ }^{1}$ College of Chemistry and Chemical Engineering, Xi'an Shiyou University, Xi'an Shaanxi 710065;

${ }^{2}$ Shaanxi Province Key Laboratory of Environmental Pollution Control and Reservoir Protection Technology of Oilfields, Xi'an, 710065;

${ }^{3}$ State Key Laboratory of Petroleum Pollution Control, CNPC Research Institute of Safety and Environmental Technology, Beijing, 102206

${ }^{*}$ Corresponding author

Keywords: Comprehensive experiment, Drilling fluids, Fracturing backflow.

\begin{abstract}
To enhance the comprehensive experiment ability and provide environmental education, an applied chemistry comprehensive experiment of preparing drilling fluid by fracturing backflow was designed in this paper. This experiment combines the basic oilfield chemicals theory, test skills and oilfield practice, which can improve students' professional test skills, skills of analysis and problem-solving. Using fracturing backflow as experimental material can improve students' environmental awareness and help to put the environmental ideas into oil production in the future.
\end{abstract}

\section{环境教育一以瓜胶压裂返排液配制钻井液实验为例}

陈刚 ${ }^{1,}{ }^{*}$, 张凡 $^{1}$, 程超 ${ }^{1}$, 张洁 $^{1}$, 薛丹 ${ }^{1,2}$, 陈世军 ${ }^{1,3}$

1西安石油大学化学化工学院, 西安 710065 , 陕西, 中国

2陕西省油气田环境污染控制技术与储层保护重点实验室, 西安 710065, 陕西, 中国

3石油石化污染物控制与处理国家重点实验室, 中国石油安全环保技术研究院, 北京, 102206, 中国 *通讯作者

关键词: 综合实验; 钻井液; 压裂返排液

摘要：为了进一步提高本科生的综合实验技能，渗透环境教育，设计了应用压裂返排液配制 钻井液的应用化学综合实验。该实验能够将油田化学理论基础与实验技术、现场实践相结合, 能够提高学生的专业实验技能、分析和解决问题的能力, 同时采用压裂返排液作为实验原料, 践行绿色环保实验教学理念, 有助于利用环保理念指导日后油田清洁生产工作。

\section{1. 引言}

压裂技术是油气井增产、注水井增注的一项重要工艺措施, 压裂过程能够形成具有高导 流能力的裂缝, 改善储层流体向井筒内流动的能力, 从而提高油气井的产能 ${ }^{[1]}$ 。当压裂结束 后产生的压裂返排液返排至地面, 压裂返排液中有700多种物质 ${ }^{[2]}$, 包括了原有的稠化剂、交 联剂、杀菌剂、稳定剂等十几种添加剂外, 还有从地层中携带的固悬物、微生物、金属离子 等, 其具有高COD、高TDS、高TSS、水质复杂特点, 其处理难度非常大, 而这些污水随意 排放会污染周边水源、庄稼农作物和土壤环境等 ${ }^{[3-4]}$ 。这与我国提倡的绿色发展、循环发展、 和谐发展背道而驰, 而将返排液处理后重复利用配制钻井液, 既可以保留返排液中的有效成 
分，也可以节约水资源，对环境保护和节约水资源都具有重要的意义 ${ }^{[5]}$ 。对石油类相关专业 学生而言, 《油田化学》就是一门必不可少的专业课。《油田化学》要求学生在理解理论的 同时, 与实践, 现场相结合, 培养学生的综合分析问题能力和解决问题的能力以及协作能力 [6]。为了使学生在有限的实验时间内掌握到更多关于该领域的知识和技能, 需要巧妙地设计 综合性实验。

环境教育是以人类与环境的关系为核心，以解决环境问题和实现可持续发展为目的，以 提高人们的环境意识和有效参与能力、普及环境保护知识与技能、培养环境保护人才为任务, 以教育为手段而展开的一种社会实践活动过程。本教研室结合科研成果设计了系列具备环保 教育特性的综合实验。综合性体现在该实验的步骤包括瓜胶基压裂液的配制、破胶、钻井液 的配制, 护胶剂的使用、压裂破胶液配制钻井液的性能评价（ $\mathrm{pH}$ 、滤失性、流变性、抑制性、 润滑性、密度）。环保性、资源节约性体现在返排液中有效成分的循环使用、水资源的循环 使用, 避免了返排液返排至地面造成的环境污染, 对返排废液的二次利用, 具有重要的环保 意义。本实验设计不仅能够使学生学习压裂液和钻井液的相关知识及 $\mathrm{K}^{+}, \mathrm{NH}_{4}{ }^{+}$对粘土水化膨 胀的影响机理, 同时又培养了学生的环保意识, 也体现了各行业倡导的环保理念 ${ }^{[7]}$ 。

\section{1. 实验目的}

（1）掌握配制瓜胶基压裂液和钻井液的基本方法;

(2) 了解钻井液处理剂的作用机理;

(3) 掌握六速旋转粘度计、滤失仪、密度计、电导率仪、 $\mathrm{pH}$ 计粘滞系数测定仪、漏斗 粘度计等主要仪器的使用方法;

（4）学会通过数据分析、资料查询等方式分析钻井液主要性能参数变化的原因。

\section{2. 实验原理}

压裂返排液作为油气开发的主要污染物之一，处理后外排难度大，处理成本高; 处理后 回注地层, 运行费用较高, 前期投入大, 且受回注地层条件的限制。而返排液处理后回用配 制钻井液, 既充分利用返排液中残余的各种添加剂和水资源, 还能有效地解决环境污染。对 于瓜胶压裂返排液中对钻井液基浆中粘土水化分散起抑制性的 $\mathrm{NH}_{4}{ }^{+}$和 $\mathrm{K}^{+}$, 通过硫酸亚铁和 $\mathrm{NH}_{4}{ }^{+}$生成复合盐对其进行调控; 通过纤维素和淀粉等高聚物维护钻井液体系, 稳定胶体, 防 止 $\mathrm{K}^{+}$进入钻井液中进行离子交换破坏钻井液体系, 从而实现瓜胶压裂返排液重复利用配制钻 井液。硫酸亚铁与铵根离子在水溶液中反应:

$$
\mathrm{Fe}^{2+}+2 \mathrm{NH}_{4}^{+}+2 \mathrm{SO}_{4}{ }^{2-}+6 \mathrm{H}_{2} \mathrm{O} \rightarrow\left(\mathrm{NH}_{4}\right)_{2} \mathrm{SO}_{4} \cdot \mathrm{FeSO}_{4} \cdot 6 \mathrm{H}_{2} \mathrm{O}
$$

硫酸亚铁溶液与等物质量铵根离子在水溶液中生成复盐硫酸亚铁铵, 硫酸亚铁铵比较稳 定, 它的六水合物 $\left(\mathrm{NH}_{4}\right)_{2} \mathrm{SO}_{4} \cdot \mathrm{FeSO}_{4} \cdot 6 \mathrm{H}_{2} \mathrm{O}$ 不易被空气氧化。

\section{3. 仪器与试剂}

羟丙基瓜尔胶、四嗍酸钠、过硫酸铵、膨润土、HV-CMC、CMC、黄原胶、淀粉、氯化 钾、碱木素、聚合氯化铝; $1000 \mathrm{~mL}$ 量筒、塘瓷桶、高速搅拌器、可调速搅拌器、天平 $(0.01 \mathrm{~g})$ 、 N P-01A 型页岩膨胀测试仪、压力机等。

\section{4. 实验步骤}

\section{1 基浆的配制}

$4 \%$ 基浆的配制: 用 $1000 \mathrm{~mL}$ 量筒量取 $3 \mathrm{~L}$ 的清水, 至于塘瓷桶中, 加入 $6 \mathrm{~g}$ 碳酸钠, 加入 $120 \mathrm{~g}$ 的钙基膨润土，边加边搅拌，加完后再摚拌两小时，放置24小时后使用。 


\section{2 钻井液的配制}

用量筒量取 $350 \mathrm{~mL} 4 \%$ 的基浆, 加入 $0.35 \mathrm{~g}$ 淀粉, 边加边搅拌, 待淀粉溶解后, 测量其密度, 粘度和滤失。

\section{3 瓜胶压裂返排液配制钻井液}

\subsection{1 瓜胶压裂液返排液的配制}

(1) 称取 $1.2 \mathrm{~g}$ 瓜胶, 缓慢加入 $400 \mathrm{~mL}$ 搅拌的水中, 搅拌均匀后, 放置 $4 \mathrm{~h}$ 以上使其充分溶 胀。

(2) 称取 $1 \mathrm{~g}$ 四嗍酸钠加入 $20 \mathrm{~mL}$ 水中, 加热使其溶解。取 $12 \mathrm{~mL}$ 四硼酸钠溶液加入 $400 \mathrm{~mL}$ 瓜胶溶液中, 用玻璃棒搅拌, 使其充分交联。

(3) 称取 $1 \mathrm{~g}$ 过硫酸铵溶于 $100 \mathrm{~mL}$ 水中。将 $100 \mathrm{~mL}$ 过硫酸铵加入水冻胶中后放入 $65^{\circ} \mathrm{C}$ 水浴 锅, 恒温加热 $30 \mathrm{~min}$ 后用旋转粘度计测定其在 $600 \mathrm{r} / \mathrm{min}$ 的粘度; 若粘度数值高于 $5 \mathrm{mPa} \cdot \mathrm{s}$, 则再 向水冻胶中加入 $20 \mathrm{~mL}$ 过硫酸铵, 加热, 测定其粘度, 如此循环直至破胶液粘度低于 $5 \mathrm{mPa} \cdot \mathrm{s}$, 观察并记录实验现象。

\subsection{2 返排液配制钻井液}

(1) 取 5 个 $500 \mathrm{~mL}$ 烧杯，将 $15 \mathrm{~mL} 1.2 \%$ 硫酸亚铁加到 $70 \mathrm{~mL}$ 压裂破胶液中搅拌均匀，再将 $0.02 \mathrm{~g}$ 聚合氯化铝和 $5.25 \mathrm{~g}$ 的碱木素混合均匀后加到压裂破胶液中, 然后和 $280 \mathrm{~mL} 5 \%$ 的基浆混 合均匀后分别加入五个烧杯中, 最后在 5 个烧杯中分别加入 $0.15 \%, 0.2 \%, 0.25 \%, 0.3 \%$, $0.35 \% \mathrm{HV}-\mathrm{CMC}$, 低速搅拌 $30 \mathrm{~min}$, 待其溶解后, 测量其密度、粘度、滤失量、摩阻系数、 $\mathrm{pH}$ 值和滤液抑制性。

(2) 护胶剂淀粉、黄原胶和CMC的操作使用方法和HV-CMC相同。（分组可分别进行 试验后, 实验结果进行对照)

\section{4 钻井液性能测试}

\subsection{1 钻井液密度}

钻井液密度计的使用方法如下:

(1) 将清水注入杯中齐杯口为止, 轻轻将盖旋转盖紧, 使多余清水从盖上小孔溢出, 擦 去外溢清水, 然后将刀口置于刀承上, 把游码左侧边线移到刻度 $1.00 \mathrm{~g} / \mathrm{cm}^{3}$ 位置, 如水平泡位 于中间，则仪器是准确的；否则，可在平衡柱内取出或加入重物来调整。

(2) 倒出清水, 擦干, 将待测泥浆注入杯中, 盖好杯盖, 让多余泥浆溢出, 擦净泥浆杯 周围的泥浆, 移动游码使横梁成水平状态（水平泡位于中间）, 游码左侧边线所对应刻度, 即为该泥浆的密度。

(3) 注意：对于混有空气或天然气的钻井液不能使用该仪器测定。

\section{4 .2 六速旋转粘度计的使用方法}

(1) 接通电源, 使外筒转速为600rpm。观察刻度盘是否对零 (若不对零, 可松开固定螺 钉调零后再拧紧) 、外筒是否偏摆（若偏摆, 应停机重新安装外筒）。检查调速机构是否灵 活可靠。

(2) 将刚高速摚拌过的钻井液倒入泥浆杯中至刻度线（此处钻井液的体积为 $350 \mathrm{~mL} ）$, 立即置于托盘限位孔上, 上升托盘, 使液面与外筒刻度线对齐, 拧紧托盘手轮。迅速从高速 (600rpm) 到低速 (300rpm) 依次测量。待刻度盘读数稳定后, 记录两个转速下的读数 $\Phi$ 。

（3）实验结束后, 关闭电源, 松开托盘手轮, 移开泥浆杯, 倒出泥浆。左旋卸下外转筒, 将外转桶和内筒清洗后擦干, 将外转筒安装在仪器上。

（4）粘度和切力的计算方法如下:

$$
\text { 表观粘度 } A V=\frac{1}{2} \Phi 600
$$

塑性粘度 $P V=\Phi 600-\Phi 300$ 
动切力 $Y P=\frac{1}{2}(2 \Phi 300-\Phi 600)$

4.4.3 静滤失仪的工作原理、使用方法及滤失量和 $\mathrm{pH}$ 值的测定

4.4.3.1 钻井液滤失原理

在滤失介质两端施加一定的压力差, 在压力差的作用下, 钻井液通过滤失介质发生滤失。

4.4.3.2 打气筒静滤失仪的结构和工作原理

静滤失仪是将一定量的钻井液, 注入筒状钻井液杯中, 上紧杯盖, 接通气源, 调节减压 阀将压力调至0.69MPa, 打开放气阀, 气源进入钻井液杯中。通过静滤失仪可记下滤失时间、 滤失量并可留取泥饼。

4.4.3.3 打气筒静滤失仪的使用方法

(1) 松开减压阀, 使减压阀处于关死状态, 此时无压力输出和显示, 然后关闭放空阀。 用力打气使气筒压力表达 $1 \mathrm{MPa}$ 左右, 然后顺时针旋转减压阀, 直到压力表读数为 $0.69 \mathrm{MPa}$ 。

（2）用手指堵住钻井液杯气接头小孔，倒入适量的泥浆，使液面与泥浆杯内刻度线平齐 （高度以低于密封圈1-1.5cm最好）, 放好干燥的密封圈, 铺一张干燥的滤纸, 将干燥的泥浆 杯盖盖好旋紧。然后装入三通接头并卡好, 将量筒放在泥浆杯下面, 对准出液孔。

（3）顺着 “进气” 箭头方向推通气阀杆，同时观察压力表指示。当压力表稍有下降或听 到有进气声后, 即停止操作通气阀, 开始计时并微调减压阀手柄, 使压力表指示保持为 $0.69 \mathrm{MPa}$ 。

（4）记录7.5min时收集的滤液量, 取开量筒, 逆着 “进气” 箭头方向推通气阀杆, 听到 放气声后表示泥浆杯中余气放尽, 取下泥浆杯。

（5）冲洗擦干泥浆杯、杯盖和密封圈。

4.4.3.4 钻井液滤失量的测定方法

假设钻井液的瞬时滤失量为 $0,7.5 \mathrm{~min}$ 时量筒中滤液体积的 2 倍即为API静滤失量。

4.4.3.5 钻井液 $\mathrm{pH}$ 值的测定方法

将pH试纸放到滤液中浸湿后取出, 待其颜色稳定后和标准色对照, 估计出泥浆的 $\mathrm{pH}$ 值。 注意: 此方法只有在滤液颜色较浅的水基钻井液中才可使用。

4.4.4 膨润土的线性膨胀率的测定方法 ${ }^{[8]}$

4.4.4.1 膨胀试验

(1) 称取 $8.00 \mathrm{~g}$ 在 $105^{\circ} \mathrm{C} \pm 3{ }^{\circ} \mathrm{C}$ 下烘干 $4 \mathrm{~h}$ 的粒度在 $0.15-0.044 \mathrm{~mm}$ 之间的二级膨润土, 小心倒 入装有滤纸的测筒(深度为 $\left.\mathrm{L}_{1}\right)$ 内。

(2) 轻轻震动测筒, 边震动边旋转, 使土粉分布均匀。

(3) 将塞杆插入测筒, 置于压力机上, 以 $10 \mathrm{MPa}$ 的压力加压 $5 \mathrm{~min}$ 。

（4）卸去压力, 取出塞杆, 用测深仪测量深度 $\mathrm{L}_{2}$ 。

4.4.4.2 用NP-01A 型页岩膨胀测试仪测定岩心膨胀率

(1) 接通主机电源, 预热 $15 \mathrm{~min}$ 。

(2) 把测杆孔盘插入测筒, 将测筒安装在主机的两根连杆之间, 测杆上端插入传感器连 杆。

(3) 调节传感器上的调节螺母，使数字表显示数字 0.00 。

(4) 用注射器把抑制剂溶液小心注入测筒至与测筒上端面齐平, 同时启动记录走纸开关。

(5) 待膨胀曲线的切线垂直于横坐标 (或 $1 \mathrm{~h}$ 内膨胀量不大于 $0.1 \mathrm{~mm}$ ) 时, 试验结束, 关 记录仪, 关主机电源。

（6）卸下测筒，取出岩心，将测筒及容器清洗干净、晾干，收存备用。

4.4.4.3 计算膨胀率 $\mathrm{S}_{\mathrm{r}}$

按(5)式计算膨胀率 $\mathrm{S}_{\mathrm{r}}$ :

$S_{\mathrm{r}}=\frac{R_{0}}{\Delta L} \times 100 \%$ 
$\Delta L=L_{1}-L_{2}$

式中: $\mathrm{Sr}$ 为膨润土的线性膨胀率;

$\mathrm{R}_{0}$ 为膨润土的膨胀量, $\mathrm{mm}$;

$\Delta \mathrm{L}$ 为岩心高度, $\mathrm{mm}$ 。

\section{5. 思考题}

(1) 钻井液的粘度测定前, 为什么通常都需要使用高速摚拌机进行搅拌?

(2) 测定静滤失量时, 要求先准备好工作压力, 然后再倒入钻井液后迅速加压测量, 请 结合静滤失方程进行解释。

（3）除用亚铁离子配位屏蔽 $\mathrm{NH}_{4}^{+}$外是否还有其他屏蔽剂? 试说明其屏蔽机理?

6. 总结

本实验涉及知识面广，需要综合考察钻井液多种重要性能，同时需要对钻井液添加剂的 作用原理有初步的了解。实验中既可以对钻井液的配制以及其作用性能, 也对压裂液的简单 配制和压裂返排液的成分及其处理方法有一定的了解。学生在查阅文献和设计实验过程中, 必须具有一定的分析问题和解决问题的能力, 有利于开阔学生视野, 提高科研潜能。同时本 实验用压裂返排液配制钻井液, 一方面解决了压裂返排液对油气田的污染, 另一方面可以提 高学生的环保意识。本实验以小组为单位开展, 有利于提升学生的协作能力和团队精神。

\section{致谢}

本文为陕西省教育厅科技服务地方专项（产业化培育项目）“杂多糖清洁钻井液在陕北 油田定向井中的应用”（17JF025）、陕西省21世纪初高等教育教学改革工程项目（0202001） 和西安石油大学教改重点项目的阶段性成果之一。

\section{Reference}

[1] M.J. Economides, B.P. Zhang. Reservoir stimulation measures, third ed., Pet. Ind. Press, 2002.

[2] I. Ferrer, E.M. Thurman Chemical constituents and analytical approaches for hydraulic fracturing waters. Trends Environ. Anal. Chem. 5 (2015) 18-25.

[3] D.M. Yang, H. Xia, J.M. Yuan, X.R. Xiang, B. Wang. Discussion on Treatment Methods of Fracturing Recovery Waste Water from Shale Gas Filed. Environ. Prot. Oil Gas Fields. 23 (2013) 20-22.

[4] D.M. Yang, J.M. Yuan, H. Xia. Environmental Problems and Counter Measures in Shale Gas Development Process. Environ. Eng. 31 (2013) 31-36.

[5] J. An, Y.C. Liu, M.Y. Chen. Advance on the Technology of Waste Hydrofracturing Fluid Treatment. Environ. Prot. Oil Gas Fields. 19 (2009) 48-50.

[6] G. Chen, J.J. Zhang, J. Zhang, L. Zhang. A comprehensive experiment of green oilfield chemical preparation from abundant fruit peel. J. Chem. Educ. 36 (2015) 58-60.

[7] D.J. Chen, F. Chen, Oil and Gas Field Applied Chemistry. Pet. Ind. Press, 2007.

[8] SY/T 6335-1997, Evaluation procedure of drilling fluids shale inhibitor. 